\title{
Detuning and Saturation of Superconducting Devices: Formulation and Measurements
}

\author{
Carlos Collado ${ }^{1}$, Jordi Mateu ${ }^{1}$, James C. Booth ${ }^{2}$, Juan M. O'Callaghan ${ }^{1}$ \\ ${ }^{1}$ Universitat Politècnica de Catalunya, Campus Nord, Barcelona, Spain \\ ${ }^{2}$ National Institute of Standards and Technology, Boulder, CO, USA
}

\begin{abstract}
Saturation and detuning of high temperature superconducting (HTS) resonators and filters may occur due to the inherent nonlinear properties of the superconductor. In HTS transmission lines, these nonlinear properties introduce a dependence of the inductance and resistance per unit length on the current through the line and, if the line forms a resonator, this current dependence provokes changes in quality factor and/or resonant frequency with incident power. This paper derives equations for the dependence of the quality factor and resonant frequency of a nonlinear transmission line vs. source power and relates it to the circuit parameters of a nonlinear transmission line. The equations are verified with measurements in a $3.3 \mathrm{GHz} \mathrm{YBa}_{2} \mathrm{Cu}_{3} \mathrm{O}_{7-\mathrm{x}}$ (YBCO) coplanar waveguide (CPW) resonator at $76 \mathrm{~K}$ and with simulations using harmonic balance.
\end{abstract}

Index Terms - Detuning, HTS, nonlinear transmission line, saturation, superconductor, superconducting resonator.

\section{INTRODUCTION}

The high performance of planar superconducting devices are known to be degraded by to the inherent nonlinear response of the superconducting material itself; i.e. the nonlinear Meissner effect [1]. This nonlinear effect can produce saturation, detuning, and generation of harmonics or intermodulation distortion (IMD) products. The distributed origin of the nonlinear effects in high temperature superconductors is known to be due to the current dependence of the superconducting penetration depth, which, for a quasi-TEM propagation mode, results in a nonlinear distributed inductance $L(i)=L_{0}+\Delta L(i)$ and a nonlinear distributed resistance $R(i)=R_{0}+\Delta R(i)$, where $i$ is the current through the line [2]. Determination of these nonlinear terms is important for improving our understanding of superconducting materials, for creating accurate circuit models of superconducting devices, and for developing strategies to reduce their nonlinear responses.

One of the most common techniques for characterizing the nonlinear effects in superconducting devices is to measure the power of the generated harmonic and IMD signals. This method is very sensitive and allows for the measurement of nonlinear effects with low incident power. However, this method does not differentiate between inductive and resistive nonlinear effects [3]. Phase measurements of the harmonic generation [4] or scalar broadband measurements of the IMD and harmonic distortion [3][5] can differentiate between $\Delta L(i)$ and $\Delta R(i)$ through the use of closed-form expressions to relate such measurements to circuit parameters that characterize the nonlinear transmission line. However, either phase or broadband techniques require complex measurement setup.

Another alternative consists of measuring the detuning (change in the resonant frequency $f_{0}$ ) and saturation (change in the quality factor $Q_{0}$ ) in resonators [6]. These measurements need more input power than IMD measurements, but allow the surface impedance versus input power to the device to be obtained. Unfortunately, relating the resistive and reactive terms of the surface impedance with the nonlinear distributed parameters $L(i)$ and $R(i)$ is not a trivial task.

These parameters can be obtained directly from the frequency response of the device at different input powers by performing nonlinear simulation with accurate distributed circuit models [7]. This method has been shown to be very powerful but requires software tools to perform accurate nonlinear simulations.

Here, we present a simple alternative using analytical closed-form expressions to relate the detuning and saturation occurring in superconducting resonators with the nonlinear distributed parameters $\Delta L$ and $\Delta R$.

\section{EQUIVALENT CIRCUIT AND FORMULATION}

This section details the analytical procedure to obtain the detuning and saturation occurring in a superconducting transmission line resonator. We quantify the detuning and saturation as the variation of the resonant frequency and quality factor $f_{0}\left(I_{0}\right)$ and $Q_{0}\left(I_{0}\right)$, respectively, with the current maximum $I_{0}$ in the standing wave pattern of the resonant mode. In what follows this current maximum is related with the incident power to the resonator, since this is the measurable quantity.

We analyzed $f_{0}\left(I_{0}\right)$ and $Q_{0}\left(I_{0}\right)$ in a superconducting halfwave transmission line resonator. The distributed superconducting nonlinear effects are taken into account by modelling the transmission line as many cascaded RLCG elemental segments [3], where the distributed resistance and inductance $R(i)$ and $L(i)$ depend on the total rf current $i$ flowing through the line. Assuming superconducting

* Contribution of the US government; not subject to US copyright 
intrinsic nonlinear effects [2] $R(i)$ and $L(i)$ follow a square law dependence given by

$$
\begin{aligned}
& L(i)=L_{d}+\Delta L_{2} i^{2} \\
& R(i)=R_{d}+\Delta R_{2} i^{2},
\end{aligned}
$$

where $L_{d}$ and $R_{d}$ are respectively the distributed inductance and resistance when $i=0$, and the terms $\Delta L_{2}$ and $\Delta R_{2}$ account for the strength of the inductive and resistive nonlinear effects.

\section{A. Detuning}

In a half-wave resonant transmission line the current distribution along the line is $i=I_{0} \sin (\pi z / l) \cos \left(\omega_{0} t\right)$, with $z$ going from 0 to $l$, where $l=\lambda / 2$, and $I_{0}$ is the maximum current at the middle of the resonator $(l=\lambda / 4)$. Thus, the resulting distributed inductance throughout the resonator can be written as $L\left(I_{0}\right)=L_{d}+\Delta L_{2} I_{0}^{2} \sin ^{2}(\pi z / l)$ and, under the assumption of weak nonlinear effects, the characteristic impedance $Z_{0}$ and phase constant $\beta_{0}$ of the transmission line are given by

$$
\begin{aligned}
& Z_{0}\left(I_{0}, z\right)=Z_{0}\left(1+\frac{\Delta L_{2}}{2 L_{0}} I_{0}^{2} \sin ^{2}\left(\pi \frac{z}{l}\right)\right) \\
& \beta_{0}\left(I_{0}, z\right)=\beta_{0}\left(1+\frac{\Delta L_{2}}{2 L_{0}} I_{0}^{2} \sin ^{2}\left(\pi \frac{z}{l}\right)\right) .
\end{aligned}
$$

Since the deviation in resonant frequency is dominated primarily by the phase shift constant $\beta_{0}\left(I_{0} z\right.$ ), we will not include the $Z_{0}$ power dependence on the analysis performed in this section.

To find the resonant frequency we use the equivalent circuit of Fig. 1, which corresponds to an isolated openended half-wave transmission line. At resonance, the impedance seen from the middle of the transmission line to the left $Z_{L}$ and right $Z_{R}$ should be 0 ; i.e., $Z_{L}=Z_{R}=0$. We can obtain the impedance seen from the middle of the line by cascading small segments of length $d_{z}$ with the z-dependent phase constant given by (4).

The result is

$$
Z_{L}=\frac{Z_{0}}{j \tan \frac{\beta_{0} l}{2}\left(1+\frac{l-2 d_{z}}{4 l} \frac{\Delta L_{2}}{4 L_{d}} I_{0}^{2}\right)},
$$

which, if $d_{z}$ tends to zero, simplifies to

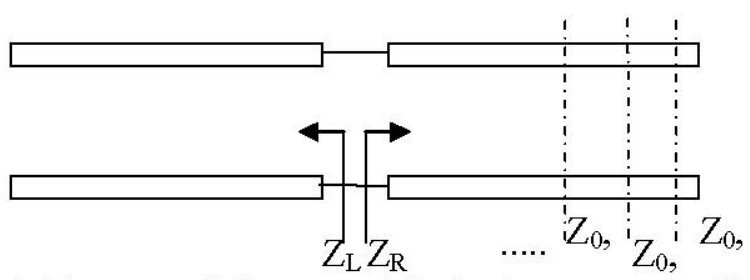

Fig. 1. The open-ended resonator is broken into segments with a nonuniform phase constant according to (4). This allows us to determine the input impedance $Z_{L}+Z_{R}$ at the centre of the line.

$$
Z_{L}=\frac{Z_{0}}{j \tan \frac{\beta_{0} l}{2}\left(1+\frac{\Delta L_{2}}{4 L_{d}} I_{0}^{2}\right)} .
$$

Now, from (6) the resonant condition including the nonlinear effects is

$$
\frac{\beta_{0} l}{2}\left(1+\frac{\Delta L_{2}}{4 L_{d}} I_{0}^{2}\right)=\frac{\pi}{2}
$$

which leads to the resonant frequency

$$
f_{0}\left(I_{0}\right)=\frac{f_{0,0}}{1+\frac{\Delta L_{2}}{4 L_{d}} I_{0}^{2}},
$$

where $f_{0,0}$ is the resonant frequency for the linear case $\left(\Delta L_{2}\right.$ $=0$ ).

This equation quantifies the detuning of the resonator due to the nonlinear distributed inductance.

\section{B. Saturation}

The unloaded quality factor $Q_{0}$ is defined as

$$
Q_{0}\left(I_{0}\right)=2 \pi f_{0}\left(I_{0}\right) \frac{\left\langle\int_{0}^{l} L_{d}(i) i^{2} d z\right\rangle}{\left\langle\int_{0}^{l} R_{d}(i) i^{2} d z\right\rangle},
$$

where the brackets $<\cdot>$ indicate time averaging.

Using (8) and (9) and taking into account the nonlinear effects on the distributed inductance $\left(L\left(I_{0}\right)=L_{d}+\Delta L_{2} I_{0}^{2} \sin ^{2}(\pi z / l)\right)$ and distributed resistance $\left(R\left(I_{0}\right)=R_{d}+\Delta R_{2} I_{0}^{2} \sin ^{2}(\pi z / l)\right)$ along the resonator, we obtain the unloaded quality factor of the superconducting resonator as

$$
Q_{0}\left(I_{0}\right)=2 \pi f_{0,0} \frac{L_{d}+\frac{1}{2} \Delta L_{2} I_{0}^{2}}{R_{d}+\frac{3}{4} \Delta R_{2} I_{0}^{2}}
$$

This equation quantifies the saturation effect that a nonlinear distributed resistance produces due to the increase in losses (decreasing quality factor) as the incident power is increased.

The resulting expressions quantifying the detuning (8) and saturation (10) show how the resonant frequency and quality factor, respectively, deviate from their linear values $\left(\Delta L_{2}=0\right.$ and $\Delta R_{2}=0$ ) as a function of the maximum current $I_{O}$ flowing in the resonator. Since $I_{O}$ is not an observable quantity, one needs to re-write (8) and (10) as a function of the available source power $P_{0}$. The section below shows how to relate $I_{0}$ and $P_{0}$ in a nonlinear superconducting resonator. Note that this cannot be done from conventional network analysis theory because it requires consideration of nonlinear distributed effects in the resonator.

\section{Maximum current in the resonator $I_{0}$}


To obtain the maximum current on the resonator we start by writing the stored energy in the resonator $W$ as [3]:

$$
W=\frac{Q_{0}\left(I_{0}\right)}{2 \pi f_{0}\left(I_{0}\right)} \frac{4 k}{(1+2 k)^{2}} P_{0},
$$

where $k$ is the coupling coefficient between the input and output port and $P_{0}$ is the actual input power on the resonator. Note that the coupling coefficient can be obtained from experimental data by use of $k=0.5\left(S_{21}\left(f_{0}\right)\right) /\left(1-S_{21}\left(f_{0}\right)\right)$.

We can also write $W$ as a function of the circuit distributed parameters and the maximum current on the resonator

$$
W=\frac{1}{4}\left(L_{d}+\frac{3}{4} \Delta L_{2} I_{0}^{2}\right) l I_{0}^{2} .
$$

Now, equating (11) and (12) and using (8) and (10) we obtain the expression

$$
16 \frac{k}{(1+2 k)^{2}} \frac{P_{0}}{R_{d} l}=I_{0}^{2}+\frac{3}{4} \frac{\Delta R_{2}}{R_{d}} I_{0}^{4} .
$$

When $\Delta \mathrm{R}_{2}=0$ the dependence between $I_{0}$ and $P_{0}$ is identical to that in a linear resonator. Otherwise, from (13) we can determine $I_{0}$ from

$$
I_{0}=\sqrt{\frac{-1+\sqrt{1+3 \frac{\Delta R_{2}}{R_{d}} 16 \frac{k}{(1+2 k)^{2}} \frac{P_{0}}{R_{d} l}}}{\frac{3}{2} \frac{\Delta R_{2}}{R_{d}}}}
$$

The combination of (14) with (8) and (10) allows for the detuning and saturation occurring in a superconducting resonator to be quantified as a function of the available incident power feeding the resonator.

\section{RESULTS}

To verify the expressions of the previous section, we modeled the resonator whose measurements are presented in

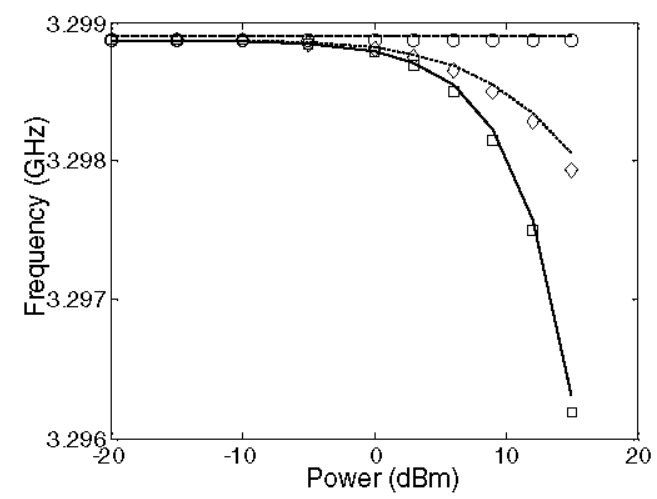

Fig.2. Detuning of a nonlinear resonator. The lines display the results of (8), (14), and the symbols are simulation results. Three sets of results are shown: resistive contribution only (dashed line and circles), inductive contribution only (solid line and squares) and inductive-resistive contribution (dotted line and diamonds). the next section. This resonator consists of a CPW halfwave resonator patterned on a $60 \mathrm{~nm}$ thick $\mathrm{YBCO}$, grown by pulsed laser deposition on a $0.5 \mathrm{~mm}$ thick $\mathrm{LaAlO}_{3}$ substrate. The cross-sectional geometry of the resonator has a central conductor with a width of $22 \mu \mathrm{m}$ and gap spacing between the central conductor and ground planes of $40 \mu \mathrm{m}$. The length is $11.33 \mathrm{~mm}$.

The circuit model consists of a cascade of 100 elemental RLCG cells [3], with (linear) distributed parameters, the distributed resistance $R_{d}$, inductance $L_{d}$, capacitance $C_{d}$ and conductance $G_{d}$ values of $21 \Omega / \mathrm{m}, 774 \mathrm{nH} / \mathrm{m}, 230 \mathrm{pF} / \mathrm{m}$ and $0 \mathrm{~S} / \mathrm{m}$, respectively (the losses of the substrate are considered negligible). Without nonlinear effects, this results in a resonant frequency of $3.3 \mathrm{GHz}$ and an unloaded quality factor of 750 .

Using the linear parameters above, we simulate three different cases: (i) nonlinear effects with a contribution from only the inductive part, with $\Delta \mathrm{L}_{2}=4.8 \times 10^{-7} \mathrm{H} /\left(\mathrm{A}^{2} \mathrm{~m}\right)$ and $\Delta R_{2}=0$; (ii) with a contribution only from the resistive part, with $\Delta \mathrm{L}_{2}=0$ and $\Delta \mathrm{R}_{2}=1 \times 10^{4} \Omega /\left(\mathrm{A}^{2} \mathrm{~m}\right)$; and (iii) nonlinear effects with contributions from both inductive and resistive parts, with $\Delta \mathrm{L}_{2}=3.4 \times 10^{-7} \mathrm{H} /\left(\mathrm{A}^{2} \mathrm{~m}\right)$ and $\Delta \mathrm{R}_{2}=7 \times$ $10^{3} \Omega /\left(\mathrm{A}^{2} \mathrm{~m}\right)$. We simulate these cases applying Harmonic Balance (HB) techniques to the equivalent circuit model describing the superconducting resonator. This method has been used and verified to characterize and predict the nonlinear response of several superconducting devices [6].

Before showing the effects of the nonlinearities on the resonant frequency and quality factor, Fig. 2 shows the resonant frequency as a function of the available power. Solid, dashed and dotted lines represent the resonance frequency obtained with (8) and (14) for cases i, ii and iii, respectively. Squares, circles and diamonds indicate respectively the resonant frequency obtained by simulating the equivalent circuit described above using $\mathrm{HB}$ techniques, for i, ii and iii. Agreement between simulation and analytical results are excellent, within $3 \%$ error, up to 15

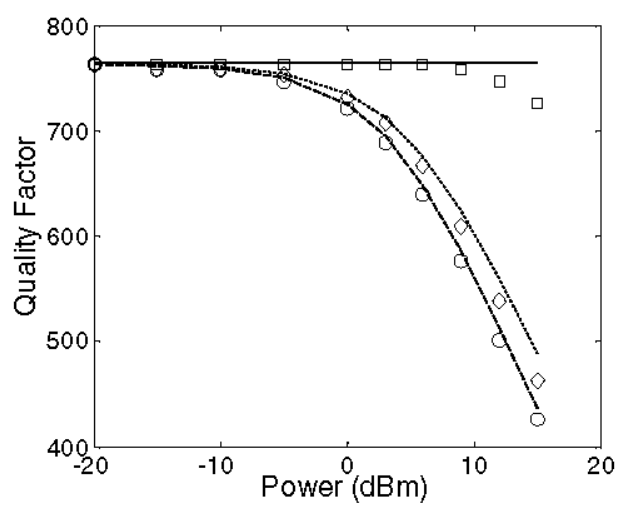

Fig.3. Saturation of a nonlinear resonator. The lines display the results of $(10),(14)$, and the symbols are simulation results. Three set of results are shown: resistive contribution only (dashed line and circles), inductive contribution only (solid line and squares), and inductive-resistive contribution (dotted line and diamonds). 


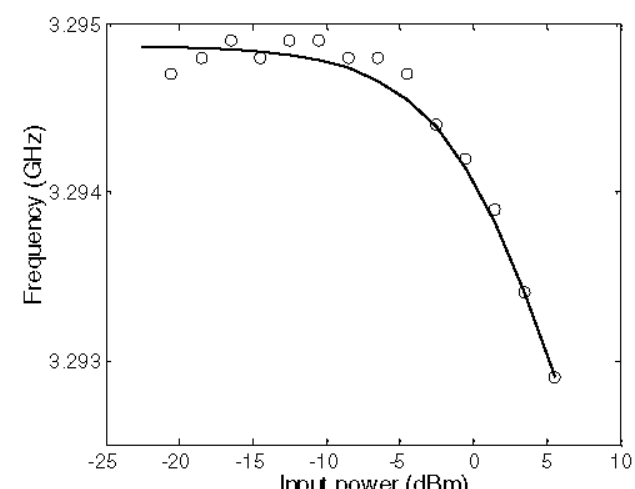

Fig.4. Resonant frequency as a function of input power. Solid line shows the best fit, using (8) (14), of the measured values (circles).

$\mathrm{dBm}$ input power.

Fig. 3 shows the unloaded quality factor. The quality factor is obtained from the simulated $\mathrm{S}_{21}$, using the common $3 \mathrm{~dB}$ method. Solid, dashed and dotted lines correspond to analytical results obtained from (10) and (14) for the cases i, ii, and iii, respectively. Simulated results are shown, respectively, as squares, circles and diamonds. Determination of the quality factor also shows good agreement between simulations and measurements for cases ii and iii. For the case of inductive nonlinearities only (i), the analytical expression predicts an almost flat behaviour, whereas the simulations indicate a reduction of $Q_{0}$ as the power increases. We believe that the method used to extract the $\mathrm{Q}_{0}$ fails when the resonance curve deviates from a Lorentzian shape [8].

In the next section, we apply simulations and closed-form expressions to determine the contribution of the nonlinear inductance $\Delta L_{2}$ and the contribution of the nonlinear resistance $\Delta R_{2}$ to the overall measured nonlinear response.

\section{MEASUREMENTS AND DisCUSSION}

Measurements of superconducting resonators were reported in [6], and consisted of power-dependent measurements of the resonator frequency response for a range of incident powers from $-20 \mathrm{dBm}$ to $6 \mathrm{dBm}$. We use the measured frequency response to obtain the resonant

I frequency and unloaded quality factor as a function of the available power. Figures 4 and 5 depict as circles the

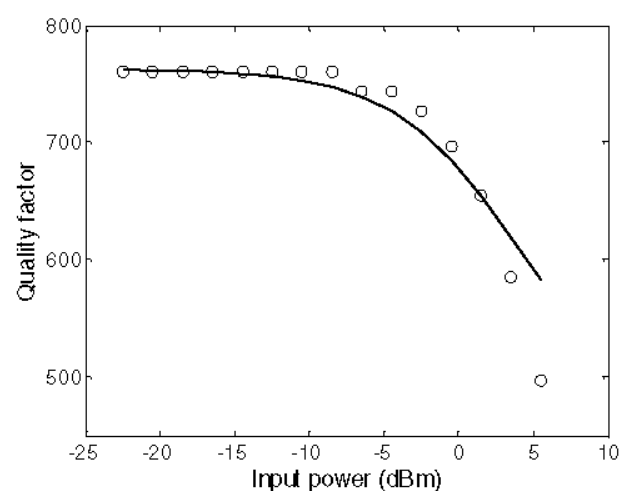

Fig.5. Unloaded quality factor as a function of the power. Solid line shows the best fit, using (10) (14), of the measured values (circles). measured $f_{0}$ and $Q_{0}$, respectively. We applied (8), (10), and (14) to extract the nonlinear inductance $\Delta L_{2}$ and nonlinear resistance $\Delta R_{2}$ that best fit the measurements. The third row in Table I details the values of $\Delta L_{2}$ and $\Delta R_{2}$. Analysis results and measured $f_{0}$ and $Q_{0}$ show good agreement over the whole power range, with only a small discrepancy in $Q_{0}$ at high powers. As mentioned previously, this may be due to the manner in which we obtained $Q_{0}$ when it deviates from the Lorentzian shape.

We compare $\Delta L_{2}$ and $\Delta R_{2}$ obtained from the closed-form equations with the ones published in [6], which were obtained through an optimization process to fit the HB simulations with the measurements. These last values are

TABLE I

SIMULATED AND EXTRACTED NONLINEAR TERMS

\begin{tabular}{lcc} 
& $\Delta \mathrm{L}_{2}\left(\mathrm{H} / \mathrm{A}^{2} \mathrm{~m}\right)$ & \multicolumn{1}{c}{$\Delta \mathrm{R}_{2}\left(\Omega / \mathrm{A}^{2} \mathrm{~m}\right)$} \\
\hline \hline HB Simulation & $1.555 \cdot 10^{-6}$ & $9.7 \cdot 10^{3}$ \\
\hline Analytical & $1.805 \cdot 10^{-6}$ & $8.5 \cdot 10^{3}$ \\
\hline \hline
\end{tabular}

detailed in the second row of Table I and show good agreement with the values of the first row. The analytical solution is therefore an efficient and fast alternative to discern between inductive and resistive nonlinear effects from simple measurements of the power dependence in superconducting resonators. Furthermore, the values of $\Delta L_{2}$ and $\Delta R_{2}$ obtained from the analytical solution are useful initial values for the HB-based fitting, which is not subject to the simplifying assumptions of the analytical approach.

\section{ACKNOWLEDGEMENT}

Work supported by the MEC Projects MAT2005-05656C04-03, TEC2006-13248-C04-02 and RYC-2005-001125.

\section{REFERENCES}

[1] D. Xu, S. K. Yip, and J. A. Sauls, "Nonlinear Meissner effect in unconventional superconductors," Phys. Rev. B, vol. 51, no. 22, pp. 16233,1995

[2] T. Dahm and D. Scalapino, "Theory of intermodulation in superconducting microstrip resonator," J. Appl. Phys., vol. 81, no. 4, pp. $2002,1997$.

[3] C. Collado, J. Mateu and J.M. O'Callaghan, "Analysis and Simulation of the Effects of Distributed Nonlinearities in Microwave Superconducting Devices" IEEE Trans. Appl. Supercond., vol. 15, no. 1, pp. 26-39, 2005

[4] J.C. Booth, K. Leong, S. A. Schima, "Phase-sensitive measurements of nonlinearity in high-temperature superconductor thin film" IEEE Trans. Appl. Supercond., vol. 15, no.2, pp.1000-1003, June 2005.

[5] J. Mateu, J. C. Booth, B. H. Moeckly, "Frequency dependence of the nonlinear response of YBCO transmission line" Appl. Phys. Letters, To be published.

[6] J. H. Oates, R.T. Shin, D.E. Oates, "A nonlinear transmission line model for superconducting stripline resonators", IEEE Trans. Appl. Supercond., vol. 3, no.1, pp.17-22, March 1993.

[7] J.C. Booth, K. Leong, S. Schima, C. Collado, J. Mateu, J. O'Callaghan, "Unified Description of Nonlinear effects in High Temperature Superconductor devices" Joumal of Supercond. DOI: 10.1007/s 10948-006-0126-2.

[8] T. Miura, "A proposal for standard to compare Q-factor evaluation accuracy of microwave resonator", 2006 IEEE MTT-S Int. Microwave Symp. Dig., pp. 1963-1966, June 2006. 